\title{
COVID-19 pandemic causing medical and public health ethical dilemmas: A case report and review of literature
}

\author{
Jarelys Hernandez, ${ }^{1}$ Barbara Lubrano di Ciccone, ${ }^{2,3}$ Sarah Thirlwell, ${ }^{4}$ Margaret Booth-Jones,,${ }^{2,3}$ Sadaf Aslam, ${ }^{1}$ John Greene ${ }^{1}$ \\ ${ }^{1}$ Department of Internal Medicine, Division of Infectious Diseases and International Medicine; ${ }^{2}$ Department of Internal Medicine, \\ Division of Hospice and Palliative Medicine, University of South Florida, Morsani College of Medicine, Tampa; ${ }^{3}$ Department of \\ Supportive Care Medicine, Division of Behavioral Medicine; ${ }^{4}$ Department of Supportive Care Medicine, Division of Palliative Medicine, \\ H. Lee Moffitt Cancer Center and Research Institute, Tampa, Florida, USA
}

\begin{abstract}
The COVID-19 pandemic presented myriad of unprecedented and daunting ethical dilemmas to healthcare workers, patients, their families, and the public health. Here we present a case of a 42-years-old Hispanic female with underlying hematological malignancy that developed severe SARS-COV-2 infection amidst the pandemic. This case illustrates some remarkable ethical dilemmas during pandemic times, including the lack of advanced directive planning, the repercussions of restricting family visits, and what ethics in crisis and moral injury entails. Identifying the ethical challenges emerging from the pandemic will assist physicians and other providers in making proper decisions and maintaining the best standard of care.
\end{abstract}

\section{Introduction}

Every epidemic may involve vexing ethical issues. Qualitative research, based on narrative, may guide the provider to afford these issues in a multidimensional con-

Correspondence: John Greene, University of South Florida, Morsani College of Medicine, 12902 USF Magnolia Drive, 33612 Tampa, FL, USA

E-mail: john.greene@moffitt.org

Key words: COVID-19; SARS-CoV-2; ethics; end of life; pandemic.

Conflict of interest: The authors declare no conflict of interest.

Availability of data and materials: All data underlying the findings are fully available.

Ethics approval and consent to participate: No ethical committee approval was required for this case report by the Department, because this article does not contain any studies with human participants or animals. Informed consent was obtained from the patient included in this study.

Consent for publication: The patient gave her written consent to use her personal data for the publication of this case report and any accompanying images.

Received for publication: 1 October 2020.

Accepted for publication: 24 November 2020.

This work is licensed under a Creative Commons Attribution NonCommercial 4.0 License (CC BY-NC 4.0).

${ }^{\circ}$ Copyright: the Author(s), 2021

Licensee PAGEPress, Italy

Qualitative Research in Medicine \& Healthcare 2021; 5:9690

doi:10.4081/qrmh.2021.9690 text for the satisfaction of all people involved. In this article we present the case of a minority woman who contracted coronavirus while immune-depressed. Her situation was hopeless since the beginning but her health care surrogate was reluctant to withdraw the life supporting system. His religious beliefs led him to consider such decision equivalent to murder. A thorough and attentive analysis of his and the patient's creed, and the intervention of other family members allowed him to accept supportive care as the most compassionate form of management of his spouse.

This case presents two novel elements with respect to the epidemics of the distant past, such as the Asian influenza of the fifties: the infection of an immune depressed patient and the availability of cardiopulmonary support. At that time the discussion of advanced directives would have been moot. As well, paying attention to the patient's culture is relatively new to medical care. In addition, through our narrative we have been able to dissect some of the personal concerns underlying acceptance of DNR (Do Not Resuscitate) orders

While all the authors participated in the management and the analysis of the case the narrative will be provided by the first author, to comply with the editorial requests of the journal.

\section{Case Report}

I was consulted for the management of a 42-years-old Hispanic female with a history of Acute Myeloid Leukemia (AML) admitted to our institution in summer of 2020. AML is an often incurable disease of the blood. Three month earlier the patient had undergone an allogeneic Bone Marrow Transplant (BMT) that appeared as 
the only potential cure. Though the leukemia had not relapsed, the patient had become more vulnerable to viral infections. To prevent the rejection and to facilitate the engraftment of the new bone marrow the patient needed to take a number of medications that suppressed her immune system.

On admission the patient was febrile, confused and short of breath. She was found to be positive by Polymerase Chain Reaction (PCR) testing to have severe acute respiratory syndrome coronavirus 2 (SARS-CoV-2) caused by the novel coronavirus disease of 2019 (COVID-19). The COVID-19 infection produced groundglass pneumonia involving both lungs. Soon the patient developed Acute Respiratory Distress Syndrome (ARDS), a condition that prevents adequate oxygenation of the blood, and distributive shock, meaning that her blood pressure was too low to maintain adequate blood flow to her vital organs, such as the brain, the heart and the kidneys. To survive, she required mechanical ventilation and administration of drugs to increase her blood pressure. She also needed intravenous fluids and a feeding tube.

On admission the patient had anemia and profound leukopenia which means a critical drop in the count of white cells with the function of fighting infection. This finding was due to the drug used to prevent the transplant rejection. Her hospital stay was complicated by the development of bilateral accumulation of air around her lungs (pneumothorax) that needed decompression with pigtail catheter placement to facilitate her respiratory function already compromised by her pneumonia. Her state of confusion kept worsening and by hospital day 4 she became unable to speak. Extensive workup of her neurological status failed to reveal any abnormality in the brain or the cerebrospinal fluid. Presumably her symptoms were due to the infection. CT of the abdomen revealed colitis and anti-toxin PCR stool testing was positive for Clostridium difficile an infection common in immune-suppressed individuals. Additionally, she was found to have reactivation herpes simplex virus-2 (HSV-2) infection around her rectum and genital area. At the time of hospitalization, visitors were not permitted due to the COVID-19 pandemic and communication with her family occurred via telephone or video conference.

The patient received the treatment available at the time for COVID-19 that included tocilizumab and remdesivir on hospital day 4 , dexamethasone on day 5 , and convalescent plasma transfusion on day 12 without clinical benefit. Her respiratory failure had worsened and she accumulated air around the heart (pneumopericardium) that impeded adequate filling of the heart. Only a surgical intervention would have allowed the removal of the air, but surgery was considered contraindicated by the patient's general condition. At the meantime the patient was seen struggling with the ventilator with teary eyes. Clearly the life-supporting system was causing a lot of distress and discomfort.
To me and to the other health professionals involved it was clear that every hope to improve the patient's condition had waned, the life supporting system was inflicting unnecessary pain on the patient. The time had come to detach the patient from the ventilator and establish palliative sedation. ${ }^{1}$ This form of treatment allows a patient in respiratory failure to pass peacefully without the so called "air hunger" one of the most disturbing symptoms of near death.

There was an impediment to palliative sedation. The patient had always refused to issue advanced directives for end of life care in at least five occasions and at the moment she was not competent to decide. Her health care surrogate by default was her spouse. He was contacted by phone by me and members of the intensive care team. He spoke a good English, albeit with an accent, and seemed to understand our consensus that any additional attempt to support his wife's life would have been not just futile, but also cruel. Yet he did not feel like to consent to the discontinuance of the ventilator and to palliative sedation. Two major themes emerged from our discussion with him "My wife is a Christian and I am a Christian," he stated "and we believe it is wrong to hasten a person's death. Every Sunday our congregation prays for Carmen's health. It would not make any sense to ask for a miracle and to let her die at the same time." "We went through so much during the past eighteen months," he added, "Carmen spent more time in the hospital than at home. When the doctor told us that her leukemia might be incurable we made a commitment to prove her wrong. We even went through a bone marrow transplant. She survived leukemia! Should she die of the flu when most people go through it untouched?" He broke out in tears. Somehow he felt cheated by the system. Was it possible that the same doctors able to cure AML should be defeated by a disease that did not seem worse than common flu?

We realized there was no much use to continue this discussion, but that it might have been wise to let the social worker explore the family situation.

We learned that the patient and her husband attended a non denominational Christian church whose pastor was a strong pro-life proponent. Carmen's parents had migrated to the US from central America. Though they had been born Catholic they found more convenient to join this church, where Carmen met her husband, an immigrant from Peru. This was a second marriage for Carmen. Apparently her first husband got killed in a car accident, though there has been some talk that he had been a victim of the drug cartel. She had been left with two children in her teens that her second husband had been happy to adopt. The present husband had had problems with alcohol and had joined the church to overcome his addiction through prayer and spiritual practices. By undertaking the responsibility of supporting Carmen and her children he found a strong enough motivation to become productive and stay. Two years ago he and Carmen had acquired the franchise of a convenience store. He had believed that 
Carmen was a personal gift from God and could not accept the idea that God could take her away from him so soon. Last but not least he was concerned about the children (20-year-old son and 17-year-old daughter). How could they survive without being able to say the last goodbye to their mother? Would have they blamed him for their mother death?

The Ethics Committee was asked to review the case and considered the clinicians' ethical concerns guided by the Four Box method to review the medical indications, patient's preferences, quality of life wishes, and contextual issues. ${ }^{2}$ The probability of recovery was deemed to be highly improbable by all the clinicians involved in her care. Further aggressive or heroic measures were considered to be futile with the risk of harm outweighing any benefits. Unfortunately, there was a lack of living will, or other advance directives, to provide information on patient's preferences and assist in the end of life discussions. It was discussed that she was only alive due to aggressive life support measures and was suffering with no quality of life.

The members of the medical Ethics team concurred with our reluctance to release a declaration of medical futility, that would have allowed us to discontinue the respiratory support without the spouse's consent. This approach might have engendered a painful confrontation with the family. Right or wrong, the perception that every effort had not been done to save Carmen's life might have disallowed husband, parents and children to come to term with her death. We ought not to load the family with guilt and resentment.

Finally, a decision was made to continue current intensive care measures without escalation of care in order to give her husband and children an opportunity to visit and provide support at the bedside. Administrative approval was requested and obtained subsequently to permit her children, spouse and parents to be at bedside in the context of limited visitation policy due to COVID-19 pandemic.

This exception to the rule proved beneficial. The family had the opportunity to say goodbye to Carmen. They also saw her crucified to the bed by the ventilator, the needles and the tubes in the futile attempt to keep her breathing. "What for this ongoing torture? Carmen is gone forever and she won't come back to us," uttered her father drowned by tears. Prior to the visit the father had been one of the staunchest supporters of indefinite continuation of life support.

The daughter walked out of the intensive care crying and sobbing: "I don't want to see my mom like this" which might be interpreted as "what have you done to my mother!" I believe this was the turning point. We were not talking anymore about a person whose survival was trusted to our ethical whims. We were talking about the beloved mother, spouse, and daughter who was suffering for no good reasons at the hand of her caregivers as directed by the family, that was ultimately responsible of her enduring pain.

In my experience, people who love a patient may rec- ognize better than any health care professional when the condition has become irreversible if given an opportunity to visit the patient. The COVID pandemic has hindered many end of life decisions because the family members did not have the opportunity to visit in person the dying beloved.

As important the visit gave us the opportunity to hold a face to face meeting instead of communicating at a distance. The family could appreciate the non-verbal message of the caregivers, and to realize they were united beyond any doubt in deeming the situation of Carmen irreversible, and they were moved by compassion and by the ethical imperative of doing no harm in recommending palliative sedation.

I found the comments of the critical care specialist particularly effective as he said: "Please remember that you don't cause Carmen's death by discontinuing the ventilator. COVID-19 is the incurable disease that causes her death. True, most people survive the COVID infection, but the situation of Carmen is special, because the cure of her leukemia also lowered her ability to fight infections. Most people survive a bee sting without any problem, but one person in ten thousand dies of a bee sting because of allergy. With respect to COVID Carmen is that one person in ten thousand." With these few direct and simple words he absolved the family of any responsibility in the death of Carmen and reassured them that she had received all available care.

In a particularly touching moment the children hugged the distraught husband and among tears they comforted him "Dad, we will be here for you with the same love mother had for you. You won't be alone."

After the meeting the family agreed without further objection to DNR (Do Not Resuscitate) and extubating orders and Carmen and Carmen expired peacefully 12 hours later.

\section{Discussion}

This case gives us the opportunity to discuss the lack of advanced care planning, ethical dilemmas related to COVID-19, and ethics in crises and moral injury. But before affording these specific issues we would like to highlight what we learned throughout our narrative. The husband and the rest of the family were adamant at the beginning in refusing the DNR order. Their attitude changed given the opportunity to visit the patient and to embrace with their heart the fact that life support represented only unnecessarily prolonged torture of their beloved spouse, mother, and daughter. Of course the anticipation of the husband's objection by the critical care specialist and the outburst of the filial love by Carmen's children that adopted their stepfather as their natural father supported the spouse in making this difficult decision.

Classical ethical or sociological research would have just stated that black and Latinx patients are less likely to 
issue advanced care directions than the Arian ones, and that religious scruples and mistrust of the system underlie the reluctance to accept a DNR order. While these aspects might have played a role in our case the strongest determinant was undoubtedly the fear of the husband to lose with his wife the strongest support in his fight with addiction as well as the first affection he could have really trusted. And perhaps there was a certain degree of anger toward the divinity that was taking her away so untimely.

In describing the reactions of the family members to the struggle of Carmen with the ventilator I highlighted how distant discussions are not substitution to the direct experience of dying, and that a face to face meeting had confirmed through non verbal signs the good intentions of the caregivers.

Last but not least the description of the case emphasized the need to observe each person primarily as a person rather than as member of a certain ethnic or cultural group. This lesson is particularly relevant to a society that is becoming more and more diverse.

\section{The ever-existent lack of Advanced Directives Planning}

It is well documented that minority populations have a lower rate of formal Advance Directives, either in the form of a Living Will or an Advance Care Plan (ACP). One factor is the identification of a more optimistic religious belief system and subsequent hopefulness that there will be a divine intervention for their loved ones. There is evidence in the literature that minority populations express a preference for more life prolonging interventions than the Arian population. Latinx are less likely to sign a DNR or allow transition to comfort measures than nonLatinx. ${ }^{3}$ Shen et al. found that when discussing ACP, Latinx preferred family involvement, acknowledgement of the religious beliefs, and a culturally competent approach with understanding of their values. ${ }^{4}$ In our case, due to the visitor restrictions, communication was limited to telephone and most frequently only involved the husband, though her father and children were known to be actively involved in our patient's care.

\section{Ethical dilemmas while caring for COVID-19 patients with surgical needs and at the end of life}

Surgical procedures on COVID-19 patients carry high mortality, ${ }^{5}$ and providers must educate and empower patients or their substitute decision-makers to make appropriate informed decisions regarding their care, including surgical interventions and other procedures. ${ }^{5}$ Compassion and transparency are urged, particularly at the end of life, ${ }^{6,7}$ in order to avoid distrust between the patient-physician relations. ${ }^{8}$ By the same token, avoiding futile interventions and alleviating suffering is an ethical imperative. ${ }^{9}$ Doctors are under no obligation to offer treatment they consider futile. ${ }^{5}$ Moreover, withholding or withdrawing of life sustaining interventions is medically appropriate course of action when interventions are deemed futile. ${ }^{7}$ Also, when surgical interventions yield similar results as non-operative treatment, the path of decreased resource utilization should be embraced. ${ }^{8}$

The restriction of visitors by the family represents also an ethical issue as our case clearly indicated.

As our institution activated its Emergency Response Plan during the COVID-19 pandemic, our resources and number of cases permitted us to maintain Emergency Management Level 1 in which conditions were serious but there was limited impact on clinical operations. However, in recognition of the growing risk of community spread and to address concerns such as providing Personal Protective Equipment (PPE) to visitors could result in diversion of resources, ${ }^{9,10}$ conditions required departure from patient-and-family-centered care practices. Due to the pandemic, administrative decisions to restrict the presence of non-essential persons were made, halting family visitation and direct participation in patient care whereas clinical teams and support staff retained their commitment to update family members regarding their loved ones' status and treatment plans.

The negative consequences related to visitor restrictions are extensive. First, the clinician's personal thoughts and emotions that arise during care allow them to provide ongoing ethical care for patients and families. ${ }^{11}$ Visitor restrictions also impair communication and the bereavement process of the family. As death occurs, the physical, mental and social consequences of physical distancing may impact the potential for complicated grief. ${ }^{11}$ Over the last decade, research has shown that Post-ICU syndrome in family members (PICS-F) is a cause of major concern. The major risk factors for PICS-F are poor communication with the ICU team, being in a decision-making role, education level, and having a loved one who died or was close to death. Indeed, many studies have shown that communication with caregivers is one of the most highly valued aspects of care that impacts family members' experience during and after the patient's stay, including the aftermath of the patient's death. Communication perceived as inconsistent, unsatisfactory or uncomforting is associated with higher risk of post-ICU burden. ${ }^{12}$ There is an increase in the rate of Post-Traumatic Stress Disorder (PTSD) in family members who did not get to say goodbye to their loved ones, further adding to complicated grieving. ${ }^{3}$ Additionally, these restrictions interfere with the rights of the patient to have family present with them for emotional support and participate in care planning during hospitalization. Visitors play a role in ensuring public scrutiny of standards of care and visitors may act as patient advocates particularly when incapacitated. ${ }^{10}$

In this case, considering the inability for the family members at the bedside, clinicians engaged the family via telephone or video conferencing to participate in care decisions. However, these efforts did not match the direct experience of being present. The challenges that exist dur- 
ing in-person communication could be further accentuated via audio or video conferencing. Specifically, in care of patients with advanced cancer, it is known that discordance exists between actual prognosis and prognostic awareness. ${ }^{13}$

\section{Ethics in crisis and moral injury}

In ideal conditions, when there is no restriction of resources and providers, medical care should be based on four ethical principles: autonomy, beneficence, non maleficence, and justice. According to autonomy the patient or his/her surrogate is the final arbiter in all medical decisions. Beneficence means that any medical intervention should be beneficial to the patient and non maleficence that the risk of treatment complications should be lower than the probability of benefits. Justice implies that there should be no discrimination based on race, sex, age, religion, or sexual preference in assigning a specific treatment.

Patient care delivery during a pandemic may require a re-defined crisis standard of care. When the demand for resources or providers overwhelms the availability it may become necessary to establish criteria to ration care. These criteria may require reserving life-saving treatment to the individuals who most may benefit from it and to those who are more beneficial to the society such as health care providers and first responders. Thus the principles of justice and beneficence may be at odds with that of autonomy and utilitarian ethics may take precedence over individual ethics. ${ }^{5,7,12}$ To honor the principle of beneficence, providers should try to relieve suffering to the best of their ability despite the intrinsic challenges of a crisis. ${ }^{5}$ Most importantly though, clinicians should resist the tendency to anticipate Level 4 crisis operations when still in Level 2-3 contingency operations in order to prevent inappropriate denial of care. ${ }^{7}$ Level 4 clinic operation mandates rationing of life-saving care.

During crisis levels, Intensive Care Unit (ICU) selection criteria are modified with patients more frequently considered mainly for palliative comfort care with the shortage of ICU beds and equipment. ${ }^{8,12}$ Ethical questions about the need for prioritization of treatment, PPE availability, testing, and resuscitation decisions arise. ${ }^{14}$ Classically, prediction of number of years to live (the use of quality-adjusted life years) is posited as the priority selection criteria. ${ }^{6,12,14}$ The risk of "sacrificing the most vulnerable patients" can result in moral injury. ${ }^{12}$ Similarly, the criteria to allocate scarce lifesaving resources like ventilators and dialysis machines may make elderly adults, people from minority communities, or people with disabilities more vulnerable. ${ }^{14}$ There is a distress in public health ethics; as we focus on saving as many lives as possible, we are inadvertently perpetuating disparities as good health is not equally distributed in the society. ${ }^{8}$ While the greater good may be prioritized during a pandemic, patient autonomy should be respected to the extent possible and clinicians should engage in advance care planning including preemptive "what if" conversations. ${ }^{15}$ Furthermore, clarification of preferences for cardiopulmonary resuscitation and other life-prolonging therapies are essential so that patient preferences can be incorporated fully into decision-making regarding allocation of scarce resources. ${ }^{16}$

Psychological effects on providers and the general population should be a concern. With patients dying mainly due to respiratory distress, and others losing human connectivity, there is rapid change and financial burden with little time to adjust. ${ }^{9}{ }^{14}$ In addition, reduced physical activity due to risk of infection compromises the patient's health and immunity. ${ }^{17}$ Those who are quarantined may experience depression, fear, guilt and anger, and those who are infected may experience anxiety, depression, guilt, stigma and anger as well. ${ }^{17}$

Fortunately we did not face a Level 4 crisis management, but the discussion is pertinent to our case. In case of rationing our patient might have been excluded from life supporting treatment based on the diagnosis of AML that is associated with a decreased life expectancy and her condition of immune-suppression that made her particularly vulnerable to COVID-19

\section{Conclusions}

This case highlights the complexity of ethical concerns surrounding the care of SARS-CoV-2 infected patients. In the case herein, health care team ensured that the care decisions were focused on the overall clinical status of the patient and were based on the principles of non-maleficence; not driven by patient COVID-19 status or fears of it. Despite inherent limitations related to the pandemic, communication and transparency were maintained.

Without a doubt, the pandemic presented a number of unprecedented challenging ethical issues. A formal crisis standard of care is needed ${ }^{14}$ including guidelines to allocate scarce resources in order to minimize or eliminate clinicians feelings that they are violating their moral beliefs. ${ }^{8}$ However, when there is minimal impact on resources, it is important to maintain usual operations in the care of patients with COVID-19 and engage more thoughtfully in advance care planning.

Identifying the ethical challenges emerging from the pandemic will assist physicians and other providers in making proper decisions and maintaining the best standard of care. ${ }^{14}$ Importantly, mental health support strategies are warranted for infected patients and their relatives, uninfected quarantined individuals, and practicing healthcare workers.

Our case demonstrates that qualitative research based on narrative may help us identify and appreciate the human elements of end of care decisions and may help shift the focus to the patient and the family from the particular ethnic and cultural group to which the patient may belong. 


\section{References}

1. Maltoni M, Scarpi E, Rosati M, et al. Palliative sedation in end-of-life care and survival: a systematic review. J Clin Oncol 2012;30:1378-83.

2. Jonsen AR, Siegler M, Winslade WJ. Clinical Ethics: A Practical Approach to Ethical Decisions in Clinical Medicine. 8th Edition ed. New York, NY: McGraw-Hills; 2015.

3. Gesi C, Carmassi C, Cerveri G, et al. Complicated Grief: What to Expect After the Coronavirus Pandemic. Front Psychiatry 2020;11:489.

4. Shen MJ, Gonzalez C, Leach B, et al. An examination of Latino advanced cancer patients' and their informal caregivers' preferences for communication about advance care planning: A qualitative study. Palliat Support Care 2020;18:277-284.

5. Harkin DW. Ethics for surgeons during the COVID-19 pandemic, review article. Ann Med Surg (Lond) 2020;55:316-9.

6. Chew C, Ko D. Medical ethics in the era of COVID-19: Now and the future. Respirology 2020;25:1033-34.

7. Kirkpatrick JN, Hull SC, Fedson S, Mullen B, Goodlin SJ. Scarce-Resource Allocation and Patient Triage During the COVID-19 Pandemic: JACC Review Topic of the Week. J Am Coll Cardiol 2020;76:85-92.

8. Dunham AM, Rieder TN, Humbyrd CJ. A Bioethical Perspective for Navigating Moral Dilemmas Amidst the COVID-19 Pandemic. J Am Acad Orthop Surg 2020;28:471-6.
9. Rosa WE, Davidson PM. Coronavirus disease 2019 (COVID19): strengthening our resolve to achieve universal palliative care. Int Nurs Rev 2020;67:160-3.

10. Coghlan N, Archard D, Sipanoun P, et al. COVID-19: legal implications for critical care. Anaesthesia 2020;75: 1517-28.

11. Wallace CL, Wladkowski SP, Gibson A, White P. Grief During the COVID-19 Pandemic: Considerations for Palliative Care Providers. J Pain Symptom Manage 2020;60:e70-6.

12. Robert R, Kentish-Barnes N, Boyer A, et al. Ethical dilemmas due to the Covid-19 pandemic. Ann Intensive Care 2020;10:84.

13. Mone S, Kerr H. Prognostic awareness in advanced cancer: an integrative literature review. BMJ Support Palliat Care 2020; bmjspcare-2020-002287. Online ahead of print.

14. Chamsi-Pasha H, Chamsi-Pasha M, Albar MA. Ethical dilemmas in the era of COVID-19. Avicenna J Med 2020;10:102-5.

15. Offodile AC, II, Aloia T. Oncology Clinical Transformation in Response to the COVID-19 Pandemic. JAMA Health Forum with JAMA Surg 2018;53:605-6.

16. Curtis JR, Kross EK, Stapleton RD. The Importance of Addressing Advance Care Planning and Decisions About DoNot-Resuscitate Orders During Novel Coronavirus 2019 (COVID-19). JAMA 2020;323:1771-2.

17. Kim SW, Su KP. Using psychoneuroimmunity against COVID-19. Brain Behav Immun 2020;87:4-5. 